\title{
A Case of Hepatic Inflammatory Pseudotumor Occurred in a Patient with Lupus Nephritis
}

\author{
Min Jung Kim ${ }^{1}$, Hyoungyoung Kim ${ }^{1}$ Yeo-Jin Song ${ }^{2}$, Soo-Kyung Cho ${ }^{1}$, Yoon-Kyoung Sung ${ }^{1}$ \\ ${ }^{1}$ Department of Rheumatology, Hanyang University Hospital for Rheumatic Diseases, ${ }^{2}$ Department of Internal Medicine, Hanyang University \\ College of Medicine, Seoul, Korea
}

\begin{abstract}
Systemic lupus erythematosus (SLE) is a systemic autoimmune disease affecting various organs. Among its manifestations, inflammatory pseudotumor (IPT) is an extremely rare disease about which no case has been reported of it occurring in the liver. We present a case of a SLE patient with hepatic IPT (hIPT) successfully treated with immunosuppressants. A 16-year-old male with elevated liver enzymes visited our clinic and was diagnosed as SLE. Although no lesion was observed in the initial abdomen ultrasonography, the abdominal CT on hospital day 7 revealed a new hepatic mass resembling an abscess. Despite 5 weeks of antibiotics treatment, the hepatic mass remained, and was re-diagnosed as hIPT secondary to SLE with an abdominal MRI. After high dose prednisolone and mycophenolate mofetil treatment, lupus activity subsided and hIPT disappeared in the follow-up CT. This case suggests that hIPT should be considered as a differential diagnosis among hepatic mass in SLE patients. (J Rheum Dis 2019;26:137-141)
\end{abstract}

Key Words. Systemic lupus erythematosus, Liver neoplasm, Granuloma, Plasma cell

\section{INTRODUCTION}

Inflammatory pseudotumor (IPT) is a rare benign neoplasm often described as a discrete, localized mass composed of a benign proliferation of inflammatory cells with fibrosis, which mimics a malignant neoplasm. Since the term "inflammatory pseudotumor" appeared in 1954, IPT has been reported in various organs, including the liver, lymph nodes, and soft tissues [1]. Its etiology and pathogenesis remain unknown.

Infection is frequently considered as main cause of IPT as antibiotic treatment cases have been reported to have contributed to the remission of IPTs [2]. IPTs were also suspected to be associated with autoimmune disorders like primary sclerosing cholangitis, rheumatoid arthritis, and Crohn's disease [3,4].

Systemic lupus erythematosus (SLE) encompasses a broad spectrum of liver diseases, from asymptomatic se- rum transaminase elevation to other autoimmune diseases such as autoimmune hepatitis or primary biliary cirrhosis. Viral hepatitis and drug-induced hepatotoxicity also cause comorbidity in SLE [5]. Reported cases of IPTs are extremely rare in SLE $[6,7]$. This paper describes an unusual case of hepatic IPT (hIPT) that occurred in a patient with active SLE.

\section{CASE REPORT}

A 16-year old male with an oral ulcer, malar rash, and polyarthralgia was referred to our hospital. At the initial evaluation, he had bicytopenia (hemoglobin $10.9 \mathrm{~g} / \mathrm{dL}$, white blood cell [WBC] $2.4 \times 10^{9} / \mathrm{L}$, eosinophil $0.012 \times 10^{9} / \mathrm{L}$ ), elevated liver enzymes (aspartate aminotransferase $132 \mathrm{U} / \mathrm{L}$, alanine aminotransferase $155 \mathrm{U} / \mathrm{L}$ ), and proteinuria (24 hours urine protein $816.4 \mathrm{mg} /$ day). Low complement level (C3 $28 \mathrm{mg} / \mathrm{dL}, \mathrm{C} 42 \mathrm{mg} / \mathrm{dL}$ ), an-

\footnotetext{
Received : July 23, 2018, Revised : October 7, 2018, Accepted : October 23, 2018

Corresponding to : Yoon-Kyoung Sung (10http://orcid.org/0000-0001-6691-8939

Department of Rheumatology, Hanyang University Hospital for Rheumatic Diseases, 222 Wangsimni-ro, Seongdong-gu, Seoul 04763, Korea. E-mail : sungyk@hanyang.ac.kr
}

Copyright (c) 2019 by The Korean College of Rheumatology. All rights reserved.

This is an Open Access article, which permits unrestricted non-commerical use, distribution, and reproduction in any medium, provided the original work is properly cited. 
ti-nuclear antibody titer $(>1: 2,560)$, anti-double-stranded DNA auto-antibody (anti-dsDNA) titer $(>1: 1,280)$, and positive anti-Smith antibody were sufficient to meet 2012 Systemic Lupus International Collaborating Clinics criteria to warrant a diagnosis of SLE with SLE Disease Activity Index score 18. Anticardiolipin antibody, lupus anticoagulant, and antineutrophil cytoplasmic antibodies (ANCA) were all negative. Initial abdomen ultrasonography revealed highly echogenic liver parenchyma and renal medullae without abnormal lesion.

On hospital day 2 , an abrupt fever $\left(38.2^{\circ} \mathrm{C}\right)$ developed, and laboratory examinations were performed. Serum inflammatory markers in the following 5 days (C-reactive protein [CRP] $69.0 \mathrm{mg} / \mathrm{L}$, erythrocyte sedimentation rate [ESR] $122 \mathrm{~mm} / \mathrm{h}$ ) showed significant elevation. However, blood gram stain, viral hepatitis lab, and cerebrospinal fluid analysis results were negative, and liver enzymes returned to normal range. To assess inflammatory focus, abdominopelvic computed tomography (APCT) was performed on hospital day 7; disclosing multiple low-density lesions on the right hepatic lobe with peripheral enhancement in the arterial phase (Figure 1). There was no abnormal finding in other organs. Based on computed tomography (CT) findings, abscess or metastatic cancer was highly suspected. However, metastatic cancer was unlikely as extrahepatic lesion was absent and the initial abdomen ultrasonography result revealed no mass like lesions. We initiated metronidazole and ceftriaxone administration, and decreased the daily dose of prednisolone from $10 \mathrm{mg}$ to $5 \mathrm{mg}$. Intravenous antibiotic treatment lasted for a total of 16 days; a follow-up CT was taken on hospital day 18, revealing unchanged hepatic mass (Figure 1). Despite negative blood culture results and lowering peak body temperature $\left(<38^{\circ} \mathrm{C}\right)$, CT findings and high ESR aroused suspicion of a hepatic pseudotumor secondary to lupus activity. On hospital day 21 , we decided to increase the prednisolone dose to $20 \mathrm{mg} /$ day, switch to oral antibiotics, and discharge the patient.

One week after being discharged, he was examined in the outpatient department. He had no fever during his discharge period, and CRP returned to normal range. However, ESR (107 mm/h) and proteinuria (urine protein/creatinine ratio [PCR] 1,043.1 mg/g) had increased. After continuing on antibiotics and prednisolone of 20 $\mathrm{mg} /$ day for another two weeks, we observed that his ESR was still high $(88 \mathrm{~mm} / \mathrm{h})$ and proteinuria had aggravated (urine PCR 1,653.8 mg/g, protein 3+, WBC 20 29/high power field $[\mathrm{HPF}]$ in urinalysis). For further work up, liver magnetic resonance imaging (MRI) was taken.

From the liver MRI, the size of the largest mass was still more than half the size of the original CT image. Lesions were slightly hypointense on T1-weighted and hyperintense on T2-weighted images. Infiltrating inflammatory tissues were seen, and intact portal veins were visible inside. The lesion had subtle high signal intensity on the axial diffusion weighted image (DWI) on high $b$-values $\left(b=800 \mathrm{~s} / \mathrm{mm}^{2}\right)$ and low signal intensity on the apparent diffusion coefficient (ADC) map, indicating mild diffuse restriction. In dynamic contrast-enhanced MRI, the lesion was isointense on portal-venous phase and showed gradual enhancement of internal septation from equilibrium to hepatobiliary phase (Figure 2). Such a radiologic pattern did not match the usual findings of a mature liver abscess, which usually presents as a high DWI signal within the necrotic abscess cavity and high ADC values at the abscess rim. Overall MRI findings suggested nonspecific inflammatory lesion mimicking hepatic mass, rather than liver abscess. As the hepatic lesion was improving after steroid administration, we proceeded with a renal biopsy instead to evaluate the worsening proteinuria. The biopsy result was consistent with type IV lupus nephritis. Since immunosuppressive treat-
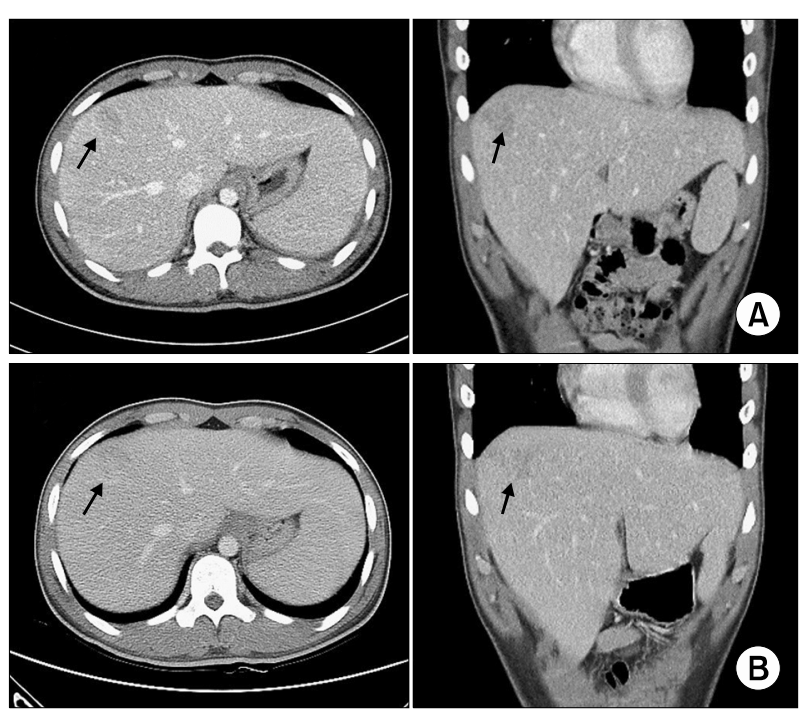

Figure 1. (A) Initial contrast enhanced abdominopelvic computed tomography (CT) on hospital day (HD) 7 with transverse (left) and coronal (right) view showing rim-enhancing hypo-attenuated lesion at subcapsular area, largest lesion (arrows) measured $3.4 \times 2.5 \mathrm{~cm}$ on segment 8 . (B) Follow-up CT on HD 18 with transverse (left) and coronal (right) view revealing unchanged hepatic lesion. 
ment was required to control active lupus nephritis, the prednisolone dosage was increased from $20 \mathrm{mg}$ to $30 \mathrm{mg}$ $(0.5 \mathrm{mg} / \mathrm{kg}) / \mathrm{day}$, and mycophenolate mofetil (MMF) 1 $\mathrm{g} /$ day was started, while antibiotics were stopped.

After changing the treatment, he remained in good condition. Follow-up APCT which was performed after 8 weeks of MMF treatment revealed complete disappearance of hepatic lesions (Figure 3). At 24 weeks after the first visit to our hospital, he was in a stable condition under a low dose $(7.5 \mathrm{mg} /$ day) of prednisolone and $2 \mathrm{~g} /$ day of MMF (Figure 4).

\section{DISCUSSION}

SLE includes various types of liver diseases. Abnormal liver function tests are seen in $23 \% \sim 55 \%$ cases of SLE patients [8]. The liver-related complications in SLE include autoimmune hepatitis, primary biliary cirrhosis, primary sclerosing cholangitis, viral hepatitis, drug in- duced hepatitis, and lymphomatous involvement of the liver [9].

Hepatic IPT, first described in 1953, usually presents with nonspecific symptoms such as fever, weight loss and general fatigue [3]. In general, hIPT is depicted as a localized mass composed of a benign proliferation of inflammatory cells with varying amounts of fibrosis or necrosis, which mimics features of an abscess or malignant neoplasm [1]. According to a multicenter study reporting 45 APCT scans of hIPT patients, $82.5 \%$ of hIPT were presented as poorly defined peripheral enhancement at the arterial phase and $77.0 \%$ as poorly defined hyperattenuating lesions with internal hypoattenuating areas at the equilibrium phase, which highly resemble typical radiological findings of liver abscess [10].

Our case reports idiopathic hepatic mass found in an adolescent male who was diagnosed as SLE. Hepatic mass formations associated with SLE activity are rare [11]. Therefore, liver biopsies were considered essential to
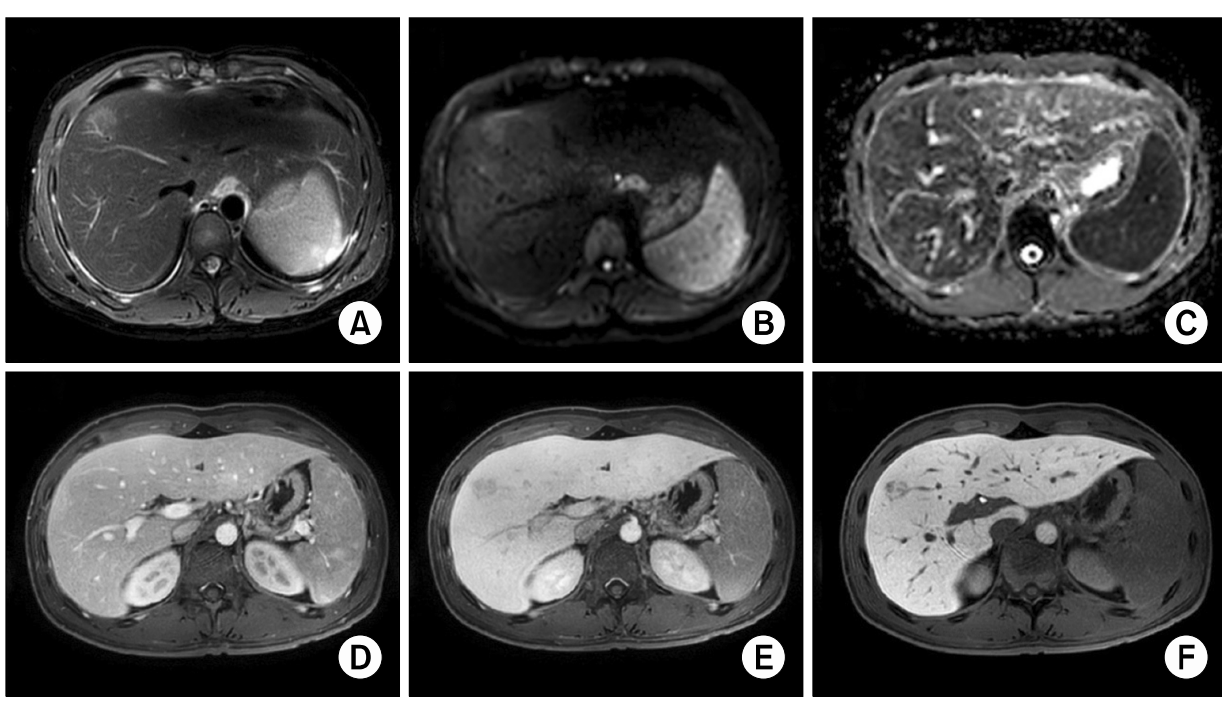

Figure 2. Follow-up transverse view of liver magnetic resonance imaging images on 5 weeks after antibiotics treatment, with (A) Axial T2-weighted fast spin echo, (B) diffusion weighted image on $b=800$ $\mathrm{s} / \mathrm{mm}^{2}$, (C) apparent diffusion coefficient map, $(D \sim F)$ portal-venous, equilibrium, and hepatobiliary phase. The largest lesion measured $2.6 \times 2.0 \mathrm{~cm}$ on segment 8.
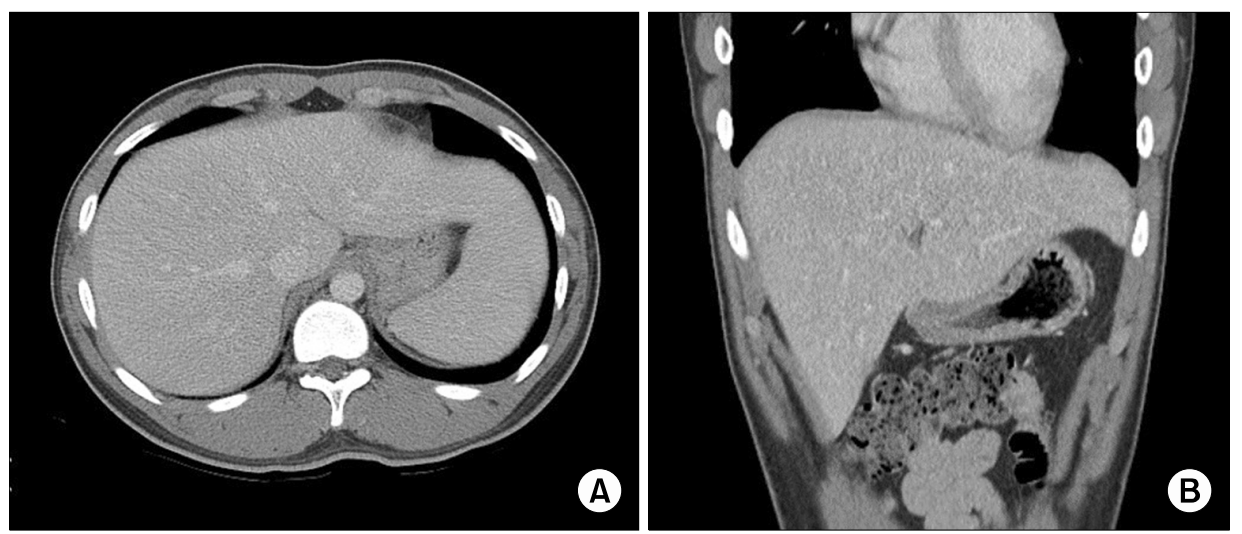

Figure 3. Follow-up of abdominopelvic contrast-enhanced computed tomography. The previous lesion on liver segment 8 was completely resolved in axial (A) and coronal view (B) of portal phase. 

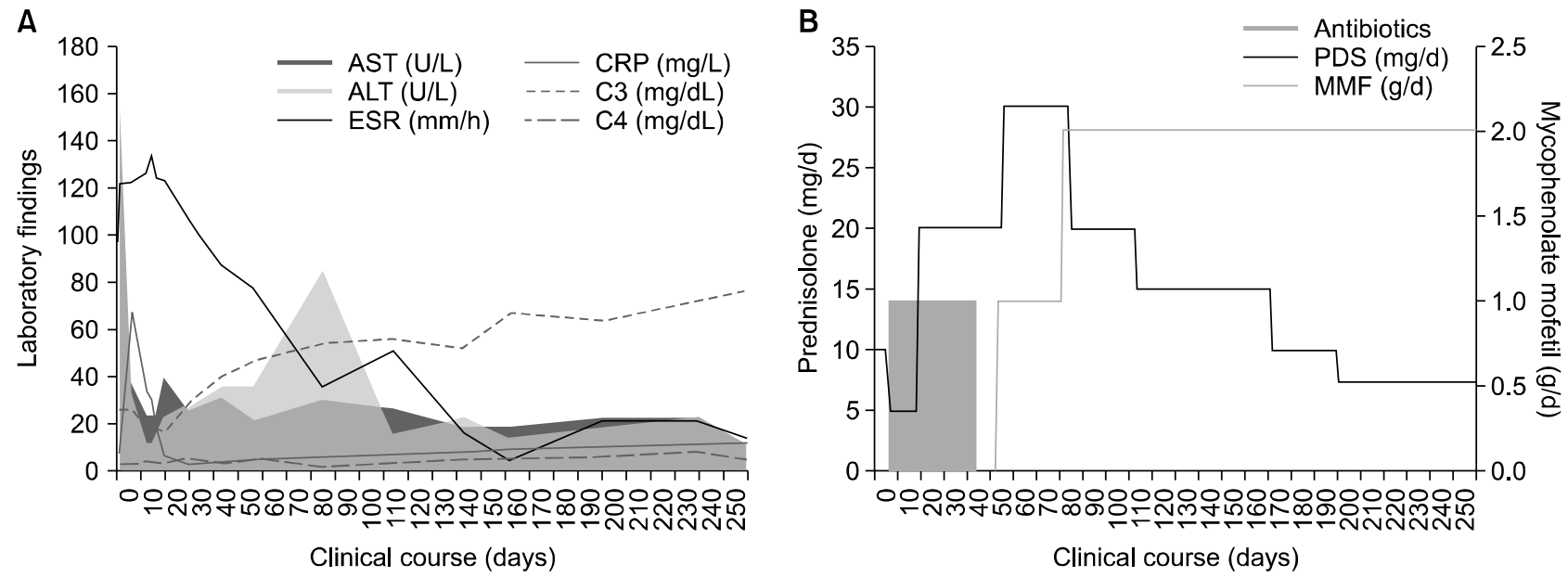

Figure 4. Clinical course of the laboratory findings (A) and the medications (B). AST: aspartate aminotransferase, ALT: alanine aminotransferase, ESR: erythrocyte sedimentation rate, CRP: C-reactive protein, C3: complement 3, C4: complement 4, PDS: prednisolone, MMF: Mycophenolate mofetil.

identify its characteristics. In our case, multiple hypodense hepatic lesions with rim enhancement on APCT, together with fever, pointed to the diagnosis of multiple liver abscesses. However, underlying SLE, negative culture results, non-response to antibiotics, high ESR, and steadily deteriorating proteinuria indicated the possibility of autoimmune related hepatic mass. The liver MRI findings helped to determine the characteristics of the hepatic mass. MRI pattens of our case differed from that of an abscess, where cellular tissues demonstrate strong intensity on higher b-value images due to restricted diffusion. Lack of necrotic core on DWI and absence of double target sign were also helpful in excluding the likelihood of liver abscess. Double target sign, the characteristic imaging feature of hepatic abscess peripheral rim, is denoted on T2-weighted images by an iso to hypointense inner layer and a hyperintense outer layer. Such MRI findings were not present in our case. Intact vasculature inside the mass even lowered possibility of liver abscess, as venous thrombophlebitis has been reported to accompany adjacent liver abscess as frequently as $42 \%$ in cases [12].

The possibility of vasculitis was also considered in this case. Vasculitis involving solely the liver in SLE has only been reported in a few published cases. Wangkaew et al. [11] reported a patient with SLE who had hepatic vasculitis with necrosis mimicking multiple liver abscesses as an initial presentation. However, it differed from our case as the lesions revealed no appreciable enhancement. Thietart et al. [13] reported a sequence of stenosis and aneurysmal dilatations of the hepatic arteries in their case report, but none of those findings corresponded to our case. Moreover, negative results of the vasculitis-related laboratory test reduced possibility of anti-phospholipid syndrome or ANCA-associated vasculitis.

Lastly, malignant tumors should be excluded. The CT findings of our case are also consistent with mass-forming Intrahepatic Cholangiocarcinoma (ICC). However, typical MRI findings of ICC such as lobulated shape, rim enhancement during the arterial phase, a target appearance with a peripheral hyperintense rim on DWI, and peripheral biliary dilatation were not found [14].

After excluding differential diseases, we diagnosed the lesion as hIPT, an inflammatory cell aggregate caused by an autoimmune entity in liver. On the MRI, a hIPT shows iso to lower signal intensity on T1-weighted images and slightly higher signal intensity on T2-weighted images. Its dynamic contrast-enhanced MRI is characterized by enhancement of the fibrous marginal zone or gradual internal septal enhancement toward the equilibrium phase [15]. In this patient, the lesion was accompanied by high lupus activity, which was totally resolved after 2 months of high dose prednisolone and MMF treatment.

To our knowledge, this is the first reported case of hIPT in SLE treated successfully with corticosteroid and MMF therapy without pathologic confirmation. In general, active liver biopsy was recommended for hIPT because it is difficult to distinguish from other diseases. However, thanks to advanced radiologic techniques, we could confidently suggest hIPT in this case without the need for invasive procedure. Based on this, we suggest that hIPT 
should be included as a major disease to be considered in SLE patients with idiopathic hepatic lesion mimicking an abscess.

\section{SUMMARY}

We report a case of an adolescent SLE patient who was diagnosed and successfully treated as having a hIPT. We should consider hIPT as a possible differential diagnosis in SLE patients with idiopathic hepatic mass and recommend the use of various radiologic techniques for its confirmation.

\section{CONFLICT OF INTEREST}

No potential conflict of interest relevant to this article was reported.

\section{REFERENCES}

1. Patnana M, Sevrukov AB, Elsayes KM, Viswanathan C, Lubner M, Menias CO. Inflammatory pseudotumor: the great mimicker. AJR Am J Roentgenol 2012;198:W217-27.

2. Ntinas A, Kardassis D, Miliaras D, Tsinoglou K, Dimitriades A, Vrochides D. Inflammatory pseudotumor of the liver: a case report and review of the literature. J Med Case Rep 2011;5:196.

3. Díaz-torné C, Narváez J, De Lama E, Diez-García M, Narváez JA, Bernad B, et al. Inflammatory pseudotumor of the liver associated with rheumatoid arthritis. Arthritis Rheum 2007;57:1102-6.

4. Younis N, Khaleeli AA, Soran H, Monteith PG. Inflamma- tory pseudotumour of the liver associated with diabetes mellitus. Int J Clin Pract 2001;55:717-9.

5. Bessone F, Poles N, Roma MG. Challenge of liver disease in systemic lupus erythematosus: clues for diagnosis and hints for pathogenesis. World J Hepatol 2014;6:394-409.

6. Khatri A, Agrawal A, Sikachi RR, Mehta D, Sahni S, Meena $\mathrm{N}$. Inflammatory myofibroblastic tumor of the lung. Adv Respir Med 2018;86:27-35.

7. Oh JS, Kwon GY, So MW, Choi SH, Kim YG, Nah SS, et al. A case of plasma cell granuloma of skull in a patient with systemic lupus erythematosus. J Korean Rheum Assoc 2006;13:311-5.

8. Piga M, Vacca A, Porru G, Cauli A, Mathieu A. Liver involvement in systemic lupus erythematosus: incidence, clinical course and outcome of lupus hepatitis. Clin Exp Rheumatol 2010;28:504-10.

9. Chowdhary VR, Crowson CS, Poterucha JJ, Moder KG. Liver involvement in systemic lupus erythematosus: case review of 40 patients. J Rheumatol 2008;35:2159-64.

10. Park JY, Choi MS, Lim YS, Park JW, Kim SU, Min YW, et al. Clinical features, image findings, and prognosis of inflammatory pseudotumor of the liver: a multicenter experience of 45 cases. Gut Liver 2014;8:58-63.

11. Wangkaew S, Lertprasertsuk N, Chotirosniramit A, Louthrenoo W. Hepatic vasculitis presenting with multiple sterile liver abscesses in a patient with systemic lupus erythematosus. Int J Rheum Dis 2007;10:64-8.

12. Syed MA, Kim TK, Jang HJ. Portal and hepatic vein thrombosis in liver abscess: CT findings. Eur J Radiol 2007;61:513-9.

13. Thietart S, Mekinian A, Delorme S, Lequoy M, Gobert D, Arrivé L, et al. Vasculitis of the hepatic artery: a case of a single-organ vasculitis. Rev Med Interne 2017;38:847-9.

14. Seo N, Kim DY, Choi JY. Cross-sectional imaging of intrahepatic cholangiocarcinoma: development, growth, spread, and prognosis. AJR Am J Roentgenol 2017;209:W64-75

15. Yan FH, Zhou KR, Jiang YP, Shi WB. Inflammatory pseudotumor of the liver: 13 cases of MRI findings. World J Gastroenterol 2001;7:422-4. 